\title{
EAE-induced upregulation of mitochondrial MnSOD is associated with increases of mitochondrial SGK1 and Tom 20 protein in the mouse kidney cortex
}

\author{
Sharanpreet Hira ${ }^{1}$ Balamuguran Packialakshmi ${ }^{1} \cdot$ Xiaoming Zhou $^{1}$ (I)
}

Received: 26 February 2019 / Accepted: 18 May 2019 / Published online: 8 June 2019

(c) This is a U.S. government work and not under copyright protection in the U.S.; foreign copyright protection may apply 2019

\begin{abstract}
Our previous demonstration that severe experimental autoimmune encephalomyelitis (EAE) increases MnSOD protein abundance in the mouse kidney cortex led this study to elucidate the underlying mechanism with monensin-treated HEK293 cells as a model. Severe EAE increases mitochondrial protein abundance of SGK1 kinase and Tom20, a critical subunit of mitochondrial translocase in the renal cortex. In HEK293 cells, catalase inhibits monensin-induced increases of mitochondrial SGK1 and Tom 20 protein levels. Further, GSK650394, a specific inhibitor of SGK1 reduces monensin-induced increase of mitochondrial protein abundance of Tom 20 and MnSOD. Finally, RNAi of Tom20 reduces the effect of monensin on MnSOD. MnSOD and Tom20 physically associate with each other. In conclusion, in HEK293 cells, mitochondrial reactive oxygen species increase protein abundance of mitochondrial SGK1, which leads to a rise of mitochondrial Tom20, resulting in importing MnSOD protein into the mitochondria. This could be a mechanism by which severe EAE up-regulates mitochondrial MnSOD in the kidney cortex.
\end{abstract}

Keywords Na,K-ATPase $\cdot$ Reactive oxygen species $\cdot$ Experimental autoimmune encephalomyelitis · Ouabain · Monensin · HEK293 cells

$\begin{array}{ll}\text { Abbreviations } \\ \text { EAE } & \text { Experimental autoimmune encephalomyelitis } \\ \text { MnSOD } & \text { Manganese superoxide dismutase } \\ \text { ROS } & \text { Reactive oxygen species } \\ \text { Tom20 } & \text { Translocase of the outer membrane subunit } 20\end{array}$

\section{Introduction}

Multiple sclerosis results from self-reactive immune cells attacking the myelin sheath in the patient's central nervous system. Using experimental autoimmune encephalomyelitis (EAE) as a model, investigators have generated a great wealth of knowledge of this autoimmune disease. In 2013,

Sharanpreet Hira and Balamuguran Packialakshmi contributed to the study equally.

Xiaoming Zhou

xiaoming.zhou@usuhs.edu

1 Department of Medicine, Uniformed Services University of the Health Sciences, 4301 Jones Bridge Road, Bethesda, MD 20814, USA
Wu et al. and Kleinewietfeld et al. first reported that a high $\mathrm{NaCl}$ diet hastens the onset and increases the severity of EAE [17, 30]. Since then, several other groups have reported the similar effects of high salt diets on EAE [10, 11, 13, 18] as well as on other types of autoimmune diseases or disease models, for example, lupus nephritis [32], rheumatoid arthritis [28] and colitis [24]. The kidney proximal tubules in the renal cortex play a critical role in the regulation of $\mathrm{Na}$ homeostasis by reclaiming approximately $65 \%$ of $\mathrm{Na}$ that is filtered through glomeruli. Na in the luminal fluid enters the proximal tubules mainly through the apical $\mathrm{Na}-\mathrm{H}$ exchanger 3 (NHE3) and exits from the tubules into blood exclusively through the basolateral Na,K-ATPase. We previously demonstrated that EAE up-regulates NHE3 and Na,K-ATPase in the kidney cortex of mice fed with a regular laboratory diet, suggesting that EAE stimulates $\mathrm{Na}$ absorption by the proximal tubules under normal salt intake [35]. Since absorption of $\mathrm{Na}$ by the renal cortex is energy dependent, we subsequently found that EAE increases the mitochondrial Complex II and Complex IV activities as well as mitochondrial reactive oxygen species (ROS) and manganese superoxide dismutase (MnSOD) activities [25]. Monensin is an ionophore that simulates $\mathrm{Na}$ absorption in the renal proximal 
tubules by stimulating $\mathrm{Na}-\mathrm{H}$ exchange and $\mathrm{Na}, \mathrm{K}$-ATPase [9]. Monensin-treated renal cells have been used as a model to study the mechanisms underlying $\mathrm{Na}$ absorption in the renal proximal tubules $[4,15]$. Using HEK293 cells as a model free of immune interference, we found that monensin increases the mitochondrial Complex II, ROS and MnSOD activities. siRNA-mediated knockdown of MnSOD impairs ATP production by mitochondria [25]. Thus, we speculate that the effect of EAE on the mitochondrial MnSOD is a protective mechanism against oxidative stress stemming from increased mitochondrial respiration in response to the energy demand from increased Na,K-ATPase activity.

MnSOD is a nucleus-encoded protein, synthesized in the cytoplasm and imported into the mitochondrial matrix. The importation of MnSOD into mitochondria is determined by the mitochondrial targeting sequence of 24 amino acids [29]. However, we and others have found that MnSOD is present in both cytosolic and mitochondrial compartments of Saccharomyces cerevisiae [22], mammalian Het-1A cells [21], HEK293 cells and mouse kidney cortex [25]. Further, we have demonstrated that a majority of MnSOD is actually localized in the cytosol of HEK293 cells and kidney cortex [25]. This observation indicates that delivery of MnSOD into mitochondria is not an automatic rather a regulated process, most likely depending on the need of mitochondria. The mitochondrial protein importation machinery is complicated. The best-characterized system is the multi-subunit TOM complex, the translocase of the outer membrane. Proteins first bind to the presequence receptors Tom20, Tom22 or Tom70 and enter the mitochondria via the pore, which is mainly formed by Tom40 [3]. Then, the TIM (translocases of the inner membrane) and SAM (sorting and assembly machinery) complexes direct the proteins to their final destination in the inner or outer membrane, the matrix or the intermembrane space [3]. The Tom 20 protein not only recognizes what protein should be imported through its sequence-specific receptors, but also determines the quantity of imported protein $[8,23]$. Emerging evidence suggests that the mitochondrial protein importation machinery is regulated by phosphorylation. For example, casein kinase phosphorylates Tom 22 at Thr57 and stimulates the assembly of Tom 22 with Tom20. In contrast, protein kinase A phosphorylates the precursor of Tom 22 at Thr76 and reduces its importation into the mitochondria [7]. However, it remains unknown whether phosphorylation is involved in Tom 20 regulation.

SGK1 is a serine/threonine kinase originally identified as a serum/glucocorticoid-induced kinase. In the kidney, SGK1 is a major stimulator of $\mathrm{Na}$ absorption by regulating almost every channel and cotransporter responsible for $\mathrm{Na}$ absorption including NHE3 and Na,K-ATPase [19, 26]. However, it is unknown whether SGK1 is involved in the regulation of MnSOD and Tom20. We previously demonstrated that EAE increases SGK1 protein abundance in the kidney cortex [35]. In present studies we first sought to determine whether EAE increases expression of the mitochondrial protein importation machinery, namely Tom 20 and Tom70, in the kidney cortex. We then used HEK293 treated with monensin as a model to examine the roles of Na,K-ATPase, mitochondrial ROS, and SGK1 in expression of Tom 20 protein and whether Tom 20 is necessary for the mitochondrial importation of MnSOD.

\section{Methods}

\section{Induction of EAE}

The male C57BL/6 mice were purchased from The Jackson Laboratory. Handling and treatment of mice were performed according to the protocol approved by Uniformed Services University IACUC. Briefly, each mouse was subcutaneously injected with PBS (control) or $100 \mu \mathrm{g}$ of $\mathrm{MOG}_{35-55}$ (New England Peptide) emulsified in $100 \mu \mathrm{l}$ complete Freund's adjuvant containing $300 \mu \mathrm{g}$ Mycobacterium tuberculosis (Fisher Scientific), and with $200 \mathrm{ng}$ pertussis toxin (List Biological Laboratories), intraperitoneally. The same dose of pertussis toxin was administered again $48 \mathrm{~h}$ later with the same route. Mice were sacrificed by $\mathrm{CO}_{2}$ inhalation followed by cervical dislocation approximately $24-48 \mathrm{~h}$ after displaying either mild (scores 1 and 2) or severe (scores 3 and 4) EAE symptoms. Control mice were sacrificed at a similar time as EAE mice. Water-replete gel food and regular pellet chow were placed on the floor of the cages in order to allow access to sick mice. The sickness was scored as 1 , flaccid tail; 2 , delay in righting reflex, hind limb weakness; 3 , flaccid paralysis in 1 hind limb; 4, flaccid paralysis in both hind limbs; and 5, quadriplegia or moribund [35].

\section{Cell culture and chemicals}

HEK293 cells were purchased from ATCC. The cells were cultured in DMEM (Sigma, D6429) plus 10\% fetal bovine serum at $37{ }^{\circ} \mathrm{C}$ supplemented with $5 \% \mathrm{CO}_{2}$. Passages of between 42 and 48 were used as inconsistent results were observed when cells beyond passage 48 were used. For the ethanol control group, the cells, at a concentration of $1 \times 10^{6} / 10 \mathrm{ml}$, were placed in 10 -cm dishes and were either siRNA-transfected or non-transfected for about $20 \mathrm{~h}$. For the monensin group, cells, at a concentration of $2 \times 10^{6} / 10 \mathrm{ml}$, were placed in 10 -cm dishes and were either siRNA-transfected or non-transfected for about $20 \mathrm{~h}$. The cells proliferated more slowly in the presence of $10 \mu \mathrm{M}$ monensin than when treated with ethanol. Then the cells were treated with $0.1 \%$ ethanol or $10 \mu \mathrm{M}$ monensin for $24 \mathrm{~h}$. In the cases of inhibitor treatments, 
the cells were preincubated with either $400 \mathrm{U} / \mathrm{ml}$ catalase (C-09322, dissolved in deionized water), $4 \mathrm{nM}$ ouabain (O3125, dissolved in deionized water) purchased from Sigma, or $2 \mu$ M GSK650394 (3572, dissolved in DMSO) purchased from Tocris Biosciences for $45 \mathrm{~min}$ before $0.1 \%$ ethanol or $10 \mu \mathrm{M}$ monensin (M5273-1G, dissolved in ethanol, Sigma) was added. The cells remained sub-confluent at the end of treatment.

\section{Transfection of cells}

The flexi-tube siRNAs against human Tom20 (GS9804) and SI00301959 were purchased from Qiagen. The control siRNA was the same as previously used [34]. The siRNAs were transfected into HEK293 cells with Lipofectamine 2000 (Thermofisher) by placing cells down simultaneously with the siRNA-Lipofectamine 2000 complex based on the recommended ratio of siRNA to Lipofectamine 2000 by the manufacturer (reverse transfection).

\section{Isolation of cytoplasm and mitochondria from the kidney and HEK293 cells}

Cytoplasmic and mitochondrial extracts from the renal cortex and HEK293 cells were isolated as previously described with slight modifications [25]. To extract the kidney cytoplasmic and mitochondrial proteins, the kidney cortex was dissected under a magnifier (Bausch and Lomb). Approximately $20 \mathrm{mg}$ of tissue from the surface region of each cortex was taken, and IB cell buffer (225 mM mannitol, $75 \mathrm{mM}$ sucrose, $0.1 \mathrm{mM}$ EGTA, $30 \mathrm{mM}$ Tris-HCl, $\mathrm{pH}$ 7.5) plus protease inhibitor tablet (Roche) was added at the ratio of $5 \mu \mathrm{lbuffer} / \mathrm{mg}$ tissue before homogenization. To extract the cytoplasmic and mitochondrial proteins from HEK293 cells, the cells were collected with a scraper after treatments and washed once with PBS, suspended in $200 \mu \mathrm{l}$ IB buffer plus protease inhibitors (Roche) and then transferred to a 5-ml glass homogenizer with an electrically powered motor (Wheaton Overhead Stirrer). Homogenization of the cortex and cells lasted $40 \mathrm{~s}$ at setting 4 . The homogenates were centrifuged at $4{ }^{\circ} \mathrm{C}$ at $600 \mathrm{~g}$ for $20 \mathrm{~min}$. The resulting pellet was discarded, while the supernatant was collected and centrifuged again at $4{ }^{\circ} \mathrm{C}$ at $10,000 \mathrm{~g}$ for $10 \mathrm{~min}$. After this centrifugation, the resulting cytosolic fraction (the supernatant) was collected and the mitochondrial fraction (the pellet) was washed once with the same buffer and centrifuged further at $4{ }^{\circ} \mathrm{C}$ at $10,000 \mathrm{~g}$ for $10 \mathrm{~min}$. The remaining pellet was then suspended in $\sim 34 \mu \mathrm{l}$ of IB buffer. A BCA assay was used to determine the protein concentrations of both cytosolic and mitochondrial extracts. After dissolved in SDS loading buffer, mitochondrial fractions were sonicated for $5 \mathrm{~s}$ to break mitochondrial DNA to facilitate loading.

\section{Western analysis}

To fraction the samples, 4-12\% Bis-Tris gel (Invitrogen) was used $(30 \mu \mathrm{g} / \mathrm{lane}$ for the kidney cortex extracts and $10 \mu \mathrm{g} /$ lane for the HEK293 extracts in most cases). The gel was transferred to a nitrocellulose membrane (Thermofisher). The membrane was submerged in a blocking buffer (Odyssey) for $1 \mathrm{~h}$ at room temperature and then probed with a primary antibody at $4{ }^{\circ} \mathrm{C}$ overnight. After a brief wash, the membrane was probed with an Alexa fluorophore conjugated secondary antibody at room temperature for $1 \mathrm{~h}$ and scanned and analyzed using infrared imaging (Li-Cor). The rabbit antibodies against SGK1 (12103S), Tom 20 (42406S), and GAPDH (2118) were purchased from Cell Signaling Technology. The rabbit MnSOD antibody (06-984) was purchased from Millipore. The mouse MnSOD antibody (MA1-106) was purchased from Invitrogen. The rabbit antibody against Tom70 (14528-1-AC) was purchased from Protein Tech, and the mouse antibody against actin (TA811000) was purchased from Origene.

\section{qPCR}

An ice-cold RNAzol RT kit (Molecular Research Center) was used to extract the total RNA from the renal cortex. The total RNA was extracted from HEK293 cells by the RNeasy Mini Kit (Qiagen). Next, the RNA was measured using NanoDrop (ThermoFisher). A High Capacity cDNA Reverse Transcription Kit (Applied Biosystems) was used to synthesize cDNA from the RNA. A SYBR Green PCR kit (Quantifast, Qiagen) was used to quantify mRNA in Stratagene Mx3005P (Agilent Technologies). The primers for the mouse Tom 20 are 5'-GCTGCAAGTGTTACA GCAGA-3' (forward) and 5'-GTCGGAAGCTTGGTC AGAAG-3' (reverse). The primers for the human Tom20 are 5'-GGAAAGGGAGCAAGGGGCAG-3' (forward) and 5'-GCCAAGTGACACCCAGCTCA-3' (reverse). The primers for the human MnSOD are 5'- CCCAATCTCCAT GATGACCTAC-3' (forward) and 5'- CACCCGATCTCG ACTGATTTAC-3' (reverse). The primers for the human SGK1 are 5' - CTTGGGCTACCTGCATTCAC-3' (forward) and 5'- GGTGGATGTTGTGCTGTTGT-3' (reverse). To measure the mRNA abundance, $200 \mathrm{ng}$ total RNA/reaction was used. mRNA was not normalized to $18 \mathrm{~s}$ rRNA and fold difference in mRNA abundance between conditions (F) was calculated as described previously [6]. 


\section{Immunoprecipitation}

After treatment, HEK293 cells were lysed in $50 \mathrm{mM}$ Tris-HCl, pH 8.0, $150 \mathrm{mM}$ sodium chloride, $1 \%$ Triton $\mathrm{X}-100$ and a protease inhibitor tablet (Roche). Protein concentration was determined with BCA (Pierce). Approximately $1 \mathrm{mg} / 200 \mu \mathrm{l}$ protein was used. Extracts were pre-cleared with mouse plain IgG (SC-2762, Santa Cruz Biotechnologies) conjugated with Protein A/G-PLUS agarose beads (SC-2003, Santa Cruz Biotechnologies) at $4{ }^{\circ} \mathrm{C}$ for $1 \mathrm{~h}$ and then incubated with mouse plain IgG- or MnSOD-protein A/G-PLUS agarose beads at $4^{\circ} \mathrm{C}$ overnight. The mouse plain IgG-agarose beads were used to determine whether IgG pulled down proteins with similar molecular weights of MnSOD and Tom20 through nonspecific binding. The agarose beads were washed twice with the lysis buffer, and then dissolved in Laemmli-SDS loading buffer. The supernatants were separated by electrophoresis in 4-12\% Bis-Tris gels (Invitrogen). Membranes were probed with rabbit anti Tom 20 and MnSOD antibodies to avoid background from mouse IgG and MnSOD antibodies and analyzed with the Odyssey infrared imager (Li-Cor).

\section{Statistical analysis}

In the analyses of mRNA and proteins from the kidney cortex, all readings were normalized to the results from the first mouse in the control groups. In the analyses of HEK293 cell studies, results were normalized to the control in each individual experiment. Data are expressed as mean \pm SEM. Statistical analyses were performed by nonpaired $t$-test, paired $t$-test, One-way ANOVA with Tukey's multiple comparisons and two-way ANOVA with Tukey's multiple comparisons, as appropriate. $P \leq 0.05$ was considered significant.

\section{Results}

\section{Severe EAE increases mitochondrial Tom 20, but not Tom70 protein abundance in the renal cortex}

We found that severe EAE increases mitochondrial Tom 20 protein abundance, whereas mild EAE does not. However, neither severe EAE nor mild EAE has a significant effect on mitochondrial Tom70 protein abundance (Fig. 1a, b). The effect of severe EAE on Tom 20 protein is apparently post-transcriptional, since it has no significant effect Tom20 mRNA level (Fig. 1c).
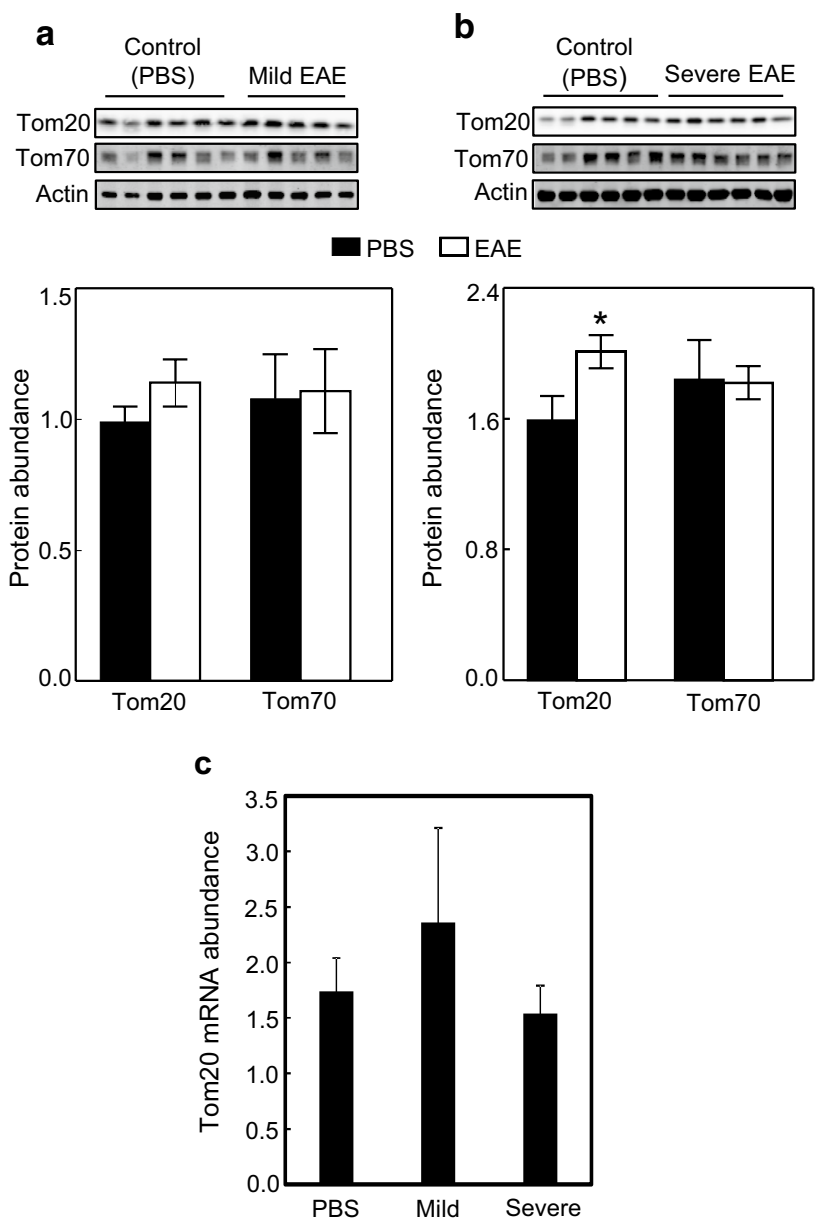

Fig. 1 Severe EAE increases Tom20, but not Tom70, protein abundance in the mitochondria of the mouse kidney cortex. a Mild EAE does not significantly increase the mitochondrial protein abundance of either Tom 20 or Tom70 in the mouse renal cortex. b Severe EAE increases the mitochondrial protein abundance of Tom20 in the extracts from the same region without significantly affecting the mitochondrial protein abundance of Tom70. EAE was induced by subcutaneous injection of $\mathrm{MOG}_{35-55}$ and scored as described in "Methods". The kidney mitochondrial proteins were extracted by homogenization followed by multiple centrifugations (see "Methods"). The extracts were separated in a 4-12\% Bis-Tris gel (Invitrogen) and probed with the rabbit anti Tom 20 and Tom 70 antibodies. c Neither severe nor mild EAE significantly changes Tom 20 mRNA levels. The total RNA from the renal cortex was extracted by ice-cold RNAzol RT kit (Molecular Research Center). Tom 20 mRNA abundance was measured by SYBR-based qPCR. (Data are expressed as mean $\pm \mathrm{SE}$ in this figure and following figures as well. $* p<0.05$ vs control; control $n=6$, mild EAE $n=5$, severe EAE $n=6$; unpaired $t$-test for $\mathbf{a}, \mathbf{b}$. Oneway ANOVA for c)

\section{Monensin elevates both Tom 20 and Tom70 protein levels, but has no significant effect on Tom 20 mRNA abundance in HEK293 cells}

Similar to the effect of severe EAE in the kidney cortex, monensin elevates Tom 20 protein level without significantly affecting its mRNA abundance (Fig. 2a, b). 


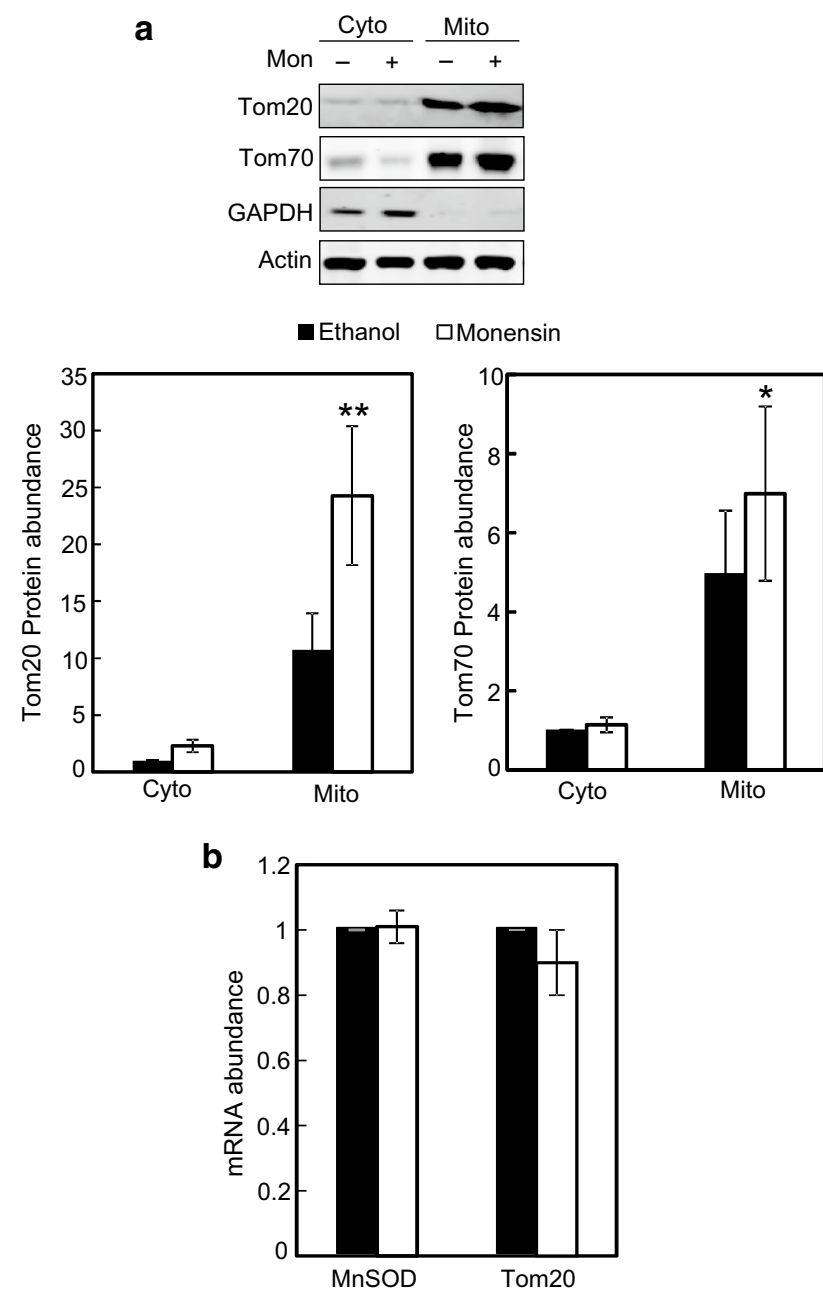

Fig. 2 Monensin increases protein abundance of Tom 20 and Tom70 in the mitochondria of HEK293 cells. a Monensin (Mon) significantly increases mitochondrial Tom 20 and Tom 70 protein abundance in the absence of a significant effect on their cytosolic protein abundance in HEK293 cells. The cells were typically placed (at a concentration of $1 \times 10^{6} / 10 \mathrm{ml}$ ) in a $10-\mathrm{cm}$ dish designated for the ethanol control group or at a concentration of $2 \times 10^{6} / 10 \mathrm{ml}$ in a $10-\mathrm{cm}$ dish designated for the monensin group, for about $20 \mathrm{~h}$. Then the cells were treated with $0.1 \%$ ethanol or $10 \mu \mathrm{M}$ monensin for $24 \mathrm{~h}$. The cytosolic (Cyto) and mitochondrial (Mito) proteins were separated by homogenization followed by multiple centrifugations and analyzed by western analysis in this figure and following figures unless indicated. GAPDH served as a marker showing adequate separation of cytosolic and mitochondrial fractions. Actin served as a loading control, but data were not normalized to it $(* p<0.05, * * p<0.01$ vs respective control; $n=6$; paired $t$-test). b Monensin has no significant effect on the mRNA abundance of MnSOD of Tom20. The cells were typically placed $\left(2 \times 10^{5} /\right.$ well $)$ in a 6 -well plate designated for the ethanol control group or $4 \times 10^{5} /$ well in a 6-well plate designated for the monensin group for about $20 \mathrm{~h}$. Then the cells were treated with $0.1 \%$ ethanol or $10 \mu \mathrm{M}$ monensin for $24 \mathrm{~h}$. The total RNA was extracted by a RNeasy Mini Kit (Qiagen), reversely transcribed and analyzed by a SYBR Green PCR kit (Quantifast, Qiagen) ( $n=6$; paired $t$-test)
However, monensin also significantly increases Tom70 protein abundance (Fig. 2a). Since EAE has no significant effect on Tom70 protein abundance in the kidney cortex, we focused our studies on Tom20.

\section{Severe EAE increases the mitochondrial SGK1 protein abundance in the kidney cortex, so does monensin in HEK293 cells. Ouabain and catalase inhibit the effect of monensin on the mitochondrial SGK1}

We previously found that severe EAE increases SGK1 protein abundance without significantly affecting its mRNA level in the kidney cortex [35]. We now show that severe EAE elevates SGK1 protein levels in the mitochondrial fraction of the region (Fig. 3a). Similarly, monensin also increases the mitochondrial SGK1 protein abundance and has no significant effect on SGK1 mRNA level in HEK293 cells (Fig. 3b-d). Ouabain, a specific inhibitor of $\mathrm{Na}, \mathrm{K}-$ ATPase, reduces the effect of monensin on mitochondrial SGK1 protein. Neither monensin nor ouabain has a significant effect on the cytosolic SGK1 protein abundance (Fig. 3b). Further, catalase almost completely eliminates the effect of monensin on the mitochondrial SGK1 (Fig. 3c). Since we previously demonstrated that severe EAE increases mitochondrial ability to generate ROS in the kidney cortex, and that monensin increases mitochondrial ROS resulting from its stimulation of Na,K-ATPase in HEK293 cells [25], we conclude that mitochondrial ROS resulted from increased $\mathrm{Na}$,K-ATPase activity that mediate the effect of monensin on mitochondrial SGK1 protein.

\section{Inhibition of SGK1 attenuates monensin-induced increases of mitochondrial MnSOD and Tom20 protein abundance}

GSK650394 inhibits SGK1 activity [27]. GSK650394 reduces monensin-induced increases of mitochondrial MnSOD and Tom20 protein levels (Fig. 4a, b). We conclude that SGK1 contributes to the effect of monensin on MnSOD and Tom20. Consistent with this conclusion is that catalase and ouabain also inhibit the effect of monensin on the mitochondrial Tom 20 protein (Fig. 4b, c). Again, as demonstrated in Fig. 2a, monensin has no significant effect on the cytosolic Tom 20 protein level (Fig. 4c). We previously showed that catalase and ouabain attenuate the effect of monensin on mitochondrial MnSOD protein abundance. We also found the same effect in the present studies (data not shown). 

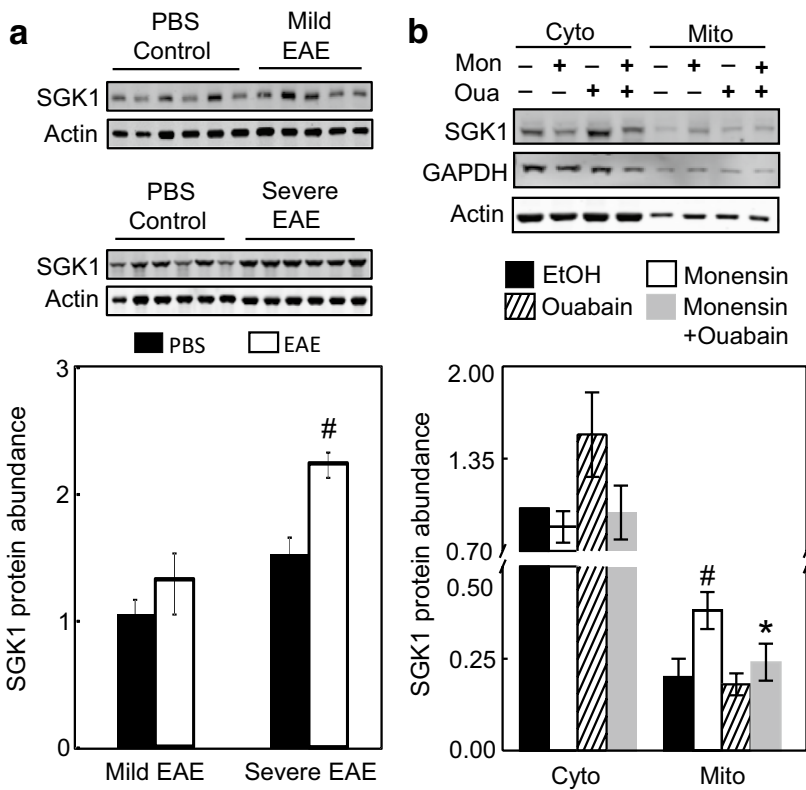

C

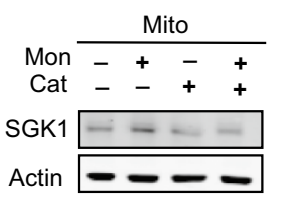

$\square$ EtOH $\square$ Monensin ZII Catalase Monensin + Catalase

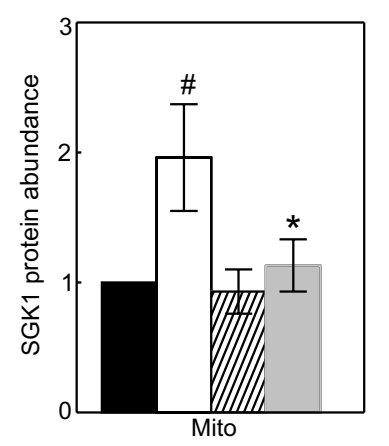

a
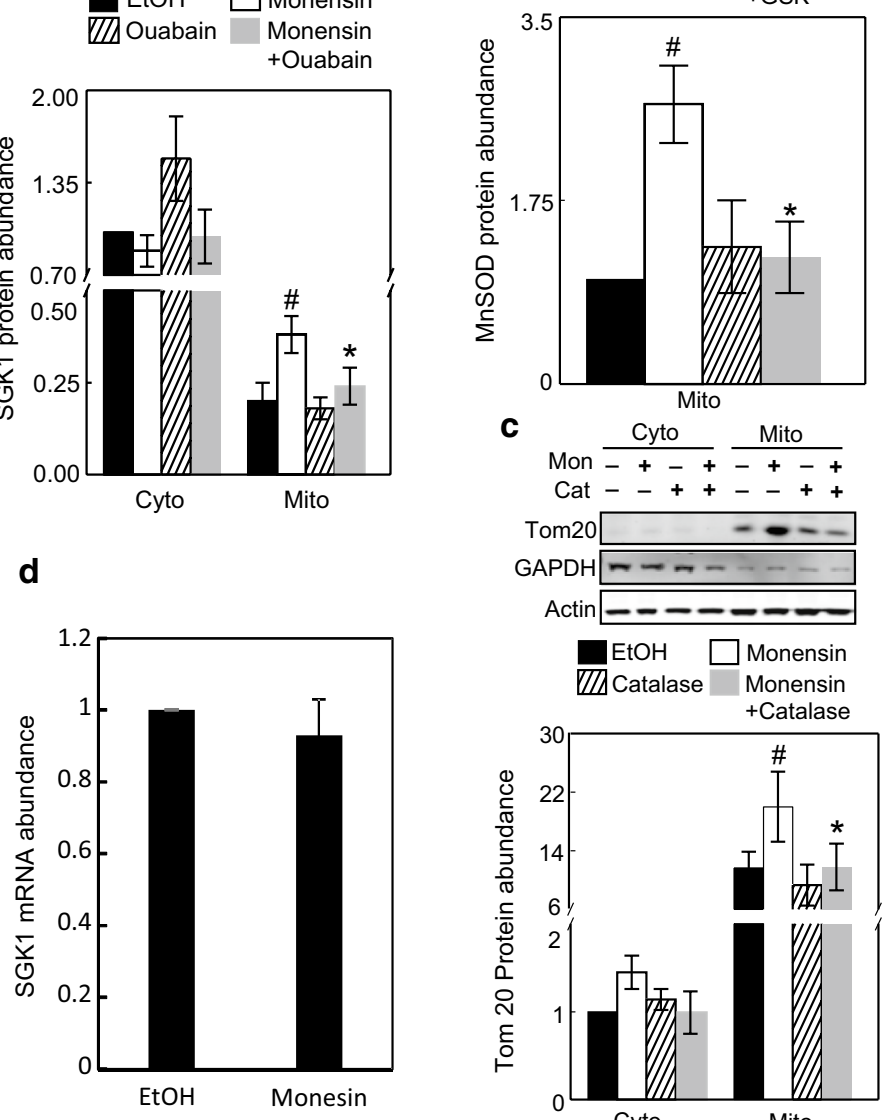

Fig. 3 Severe EAE and monensin increases SGK1 protein abundance in the mitochondria of kidney cortex and HEK293 cells, respectively. Ouabain and catalase inhibit the effect of monensin on the mitochondrial SGK1 in HEK293 cells. a Severe EAE significantly increases the mitochondrial SGK1 protein abundance, whereas mild EAE does not. The same mitochondrial extracts in Fig. 1 were used for analysis of SGK1 protein abundance $\left({ }^{\#} p<0.01\right.$ vs control; unpaired $t$-test). b Monensin (Mon) increases the mitochondrial (Mito) SGK1 protein abundance without significantly affecting the cytosolic (Cyto) one, and ouabain (Oua) inhibits the effect of monensin on the mitochondrial SGK1 in HEK293 cells. The subconfluent cells as described in Fig. 2a legend were pre-incubated with deionized water or $4 \mathrm{nM}$ ouabain for $45 \mathrm{~min}$ before $0.1 \%$ ethanol or $10 \mu \mathrm{M}$ monensin was added for an additional $24 \mathrm{~h}$ in a $10-\mathrm{cm}$ dish. $\left[{ }^{\#} p<0.01\right.$ vs the mito EtOH group (control), ${ }^{*} p<0.05$ vs the mito monensin group, $n=8$, two-way ANOVA]. c Catalase (Cat, $400 \mathrm{U} / \mathrm{ml}$ ) also inhibits the effect of monensin on the mitochondrial SGK1 protein abundance. The cells were treated in the same way as treated with ouabain $\left[{ }^{\#} p<0.01\right.$ vs the mito EtOH group (control), $* p<0.05$ vs the mito monensin group, $n=7$, two-way ANOVA]. d Monensin has no significant effect on SGK1 mRNA abundance. The subconfluent cells as described in Fig. 2b legend were treated with $0.1 \%$ ethanol (control) or $10 \mu \mathrm{M}$ monensin for $24 \mathrm{~h}$ in a 6-well plate before they were collected for mRNA analysis $(n=6$, paired $t$-test)

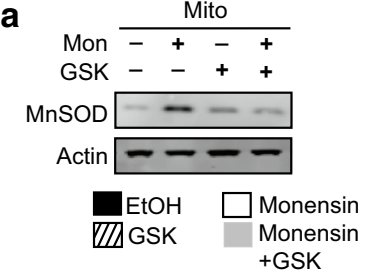

c
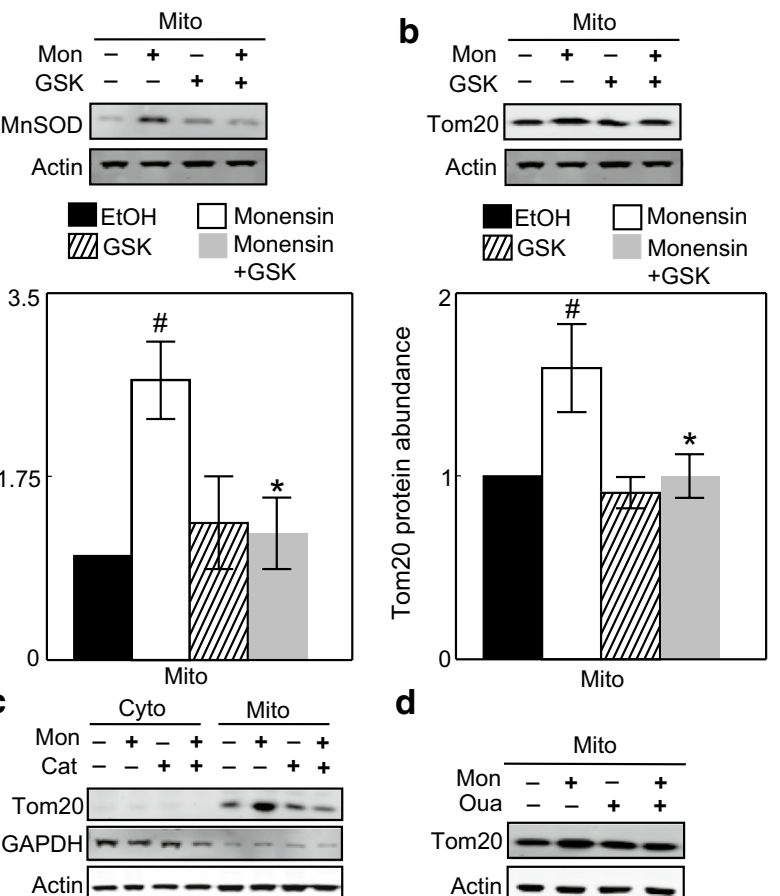

d
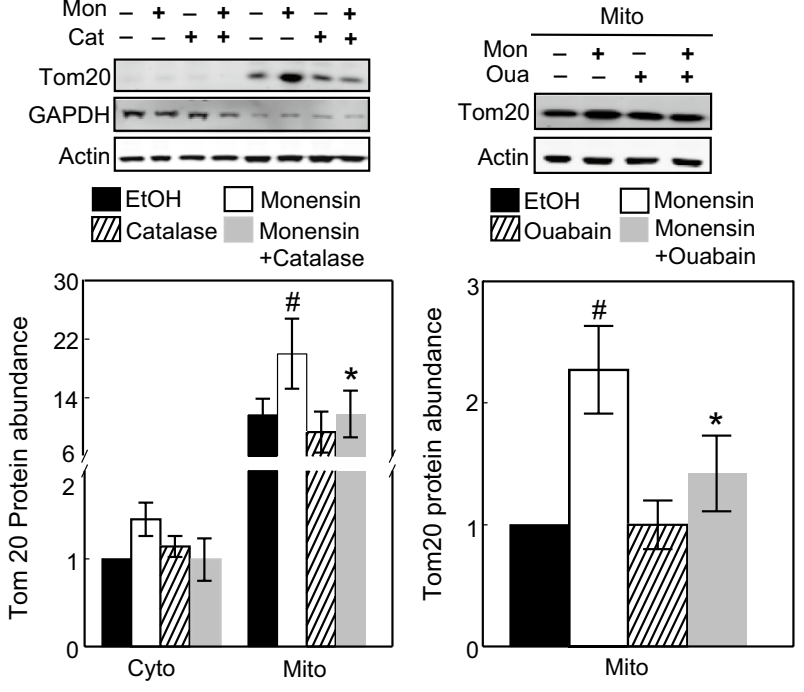

Fig. 4 GSK650394, a specific inhibitor of SGK1, reduces monensin-induced increase of mitochondrial MnSOD and Tom20 protein abundance, and catalase and ouabain blunt the effect of monensin on the mitochondrial Tom20 protein abundance in HEK293 cells. a GSK650394 significantly inhibits the effect of monensin on the mitochondrial MnSOD protein abundance. The subconfluent cells as described in Fig. 2a legend were pre-incubated with $0.1 \%$ DMSO or $2 \mu \mathrm{M}$ GSK650394 for 45 min before $0.1 \%$ ethanol (control) or $10 \mu \mathrm{M}$ monensin was added for additional $24 \mathrm{~h}\left({ }^{\#} p<0.01 \mathrm{vs}\right.$ the mito EtOH group, ${ }^{*} p<0.05$ vs the mito monensin group, $n=7$, two-way ANOVA). b GSK650394 significantly inhibits monensininduced increase of mitochondrial Tom 20 protein abundance. The HEK293 cells were treated in same way as in a. $\left({ }^{\#} p<0.01\right.$ vs the mito EtOH group, $* p<0.05$ vs the mito monensin group, $n=7$, two-way ANOVA). c, d Both catalase (400 U/ml) and ouabain (4 $\mathrm{nM})$ attenuate monensin-induced increase of mitochondrial Tom20 protein abundance. The cells were treated in the same way as in Fig. 3b, c. $\left({ }^{\#} p<0.01\right.$ vs the mito EtOH group, ${ }^{*} p<0.05$ vs the mito monensin group, in $\mathbf{c}, n=11$; in $\mathbf{d}, n=10$, two-way ANOVA for both) 
a
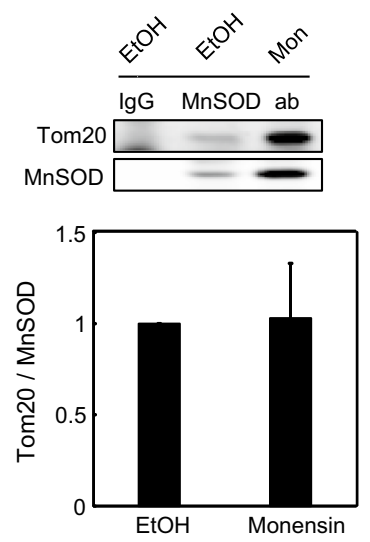

b
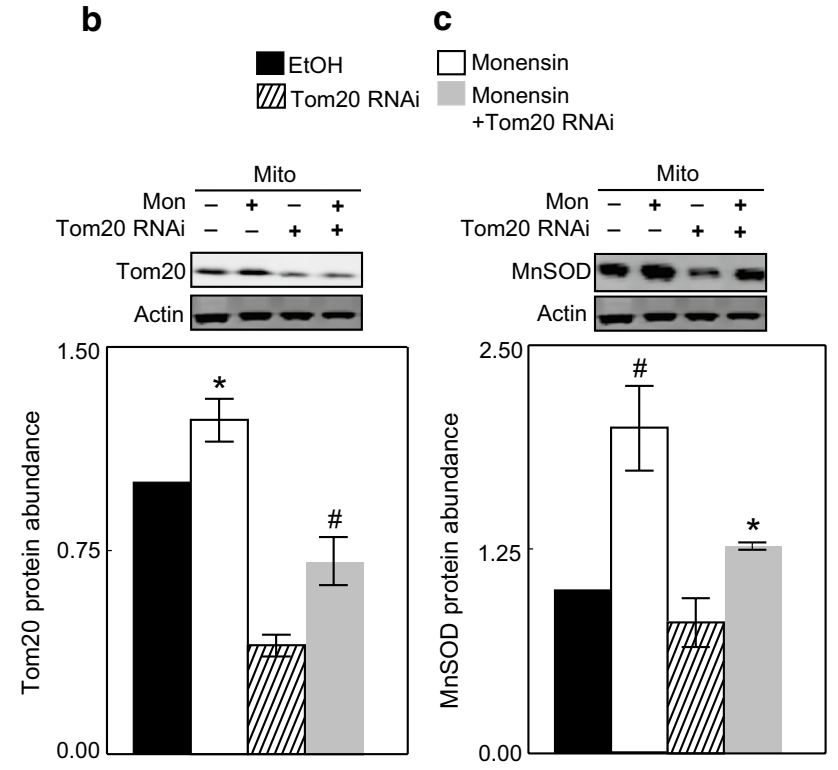

Fig. $5 \mathrm{MnSOD}$ and Tom20 physically associate with each other, and inhibition of Tom20 reduces monensin-induced increase of mitochondrial MnSOD level. a MnSOD is physically associated with Tom20. The subconfluent HEK293 cells as described in Fig. 2a legend were treated with $0.1 \%$ ethanol (control) or $10 \mu \mathrm{M}$ monensin for $24 \mathrm{~h}$ in a $10-\mathrm{cm}$ dish before they were collected with a lysis buffer. MnSOD in the total cell supernatant was immunoprecipitated with a plain mouse IgG serving as a control for non-specific binding or a mouse anti MnSOD antibody. The immunoprecipitated MnSOD and Tom20 were identified with rabbit anti MnSOD and Tom20 antibodies through western analysis $(n=3)$. b, $\mathbf{c}$ Knockdown of Tom 20 protein by its siRNA significantly reduces monensin-induced increase of mitochondrial MnSOD protein abundance. The cells were typically placed (at a concentration of $1 \times 10^{6} / 10 \mathrm{ml}$ ) in a 10 -cm dish simultaneously with $60 \mathrm{nM}$ siRNA-Lipofectamine 2000 complex designated for the ethanol control group or at a concentration of $2 \times 10^{6} / 10 \mathrm{ml}$ in a $10-\mathrm{cm}$ dish with $60 \mathrm{nM}$ siRNA-Lipofectamine 2000 complex designated for the monensin group for about $20 \mathrm{~h}$. Then the cells were treated with $0.1 \%$ ethanol or $10 \mu \mathrm{M}$ monensin for $24 \mathrm{~h}$. The mitochondrial (Mito) proteins were separated and analyzed (in b, ${ }^{*} p<0.05$ vs the mito EtOH, ${ }^{\#} p<0.001$ vs the mito monensin group; in $\mathbf{c},{ }^{\#} p<0.001$ vs the mito EtOH group, ${ }^{*} p<0.05$ vs the mito monensin group, $n=6$ and two-way ANOVA for both)
Tom20 physically associates with MnSOD, and inhibition of Tom 20 by its siRNA reduces the effect of monensin on the mitochondrial MnSOD protein

The antibody against MnSOD co-immunoprecipitated Tom20, and monensin does not significantly affect the coimmunoprecipitation ratio (Fig. 5a). We also tested whether the antibody against Tom 20 could co-immunoprecipitate MnSOD and found no evidence that the antibody specifically pulled down Tom20 (data not shown). Finally, RNAi of Tom 20 with its siRNA significantly reduces monensininduced increase of mitochondrial MnSOD protein abundance (Fig. 5b, c). We conclude that Tom 20 and MnSOD are physically associated with each other, and the increase of mitochondrial MnSOD protein abundance by monensin is dependent on Tom20.

\section{Discussion}

\section{Importation of MnSOD into mitochondria is dependent on Tom 20 in mammalian cells}

MnSOD is the first line of defense against mitochondrial oxidative stress. The significance of MnSOD is underscored by the observation showing that MnSOD knockout mice die within the first day of life due to dilated cardiomyopathy and neurodegeneration [20]. Although the majority of MnSOD protein and activity is present in the cytosol of HEK293 cells, in contrast to its counterpart in the mitochondria, the cytosolic MnSOD is not sensitive to ROS and monensin, indicating that the cytosolic MnSOD is regulated by a different mechanism [25]. We previously demonstrated that severe EAE increases the mitochondrial MnSOD protein level without any significant effect on its mRNA level, and this effect is also seen in HEK293 cells treated with monensin [25]. In the present studies, we continuously used HEK293 cells as a model to understand how monensin only elevates the mitochondrial MnSOD protein level without significantly affecting its cytosolic abundance or mRNA level (Fig. 2b) [25]. It has been demonstrated that the mitochondrial targeting signal of MnSOD directs MnSOD mRNA or a chimera mRNA containing the targeting signal to the surface of mitochondria and the mRNA is translated there, thus improving mitochondrial importation efficiency [14, 22], but this mechanism does not guarantee that the translated protein will enter mitochondria unless the importation mechanism is coordinated. Indeed, the majority of MnSOD localized in the cytosol of HEK293 [25] indicates that the mitochondrial importation rather than protein synthesis is the rate-limiting step for accumulation of mitochondrial MnSOD. Computational modeling suggests that the plant 
Oryza sativa mitochondrial SOD would favorably bind with Tom20, exhibiting the lowest binding free energy among all candidates [33]. To determine whether Tom20 is critical to mitochondrial importation of MnSOD, we first demonstrated that severe EAE elevates mitochondrial Tom20, but not Tom70, protein levels in the mouse renal cortex (Fig. 1a, b). We then found that monensin has a similar effect on Tom20 in HEK293 cells (Fig. 2a). More importantly, knockdown of Tom 20 by its siRNA attenuates the effect of monensin on mitochondrial MnSOD protein levels, and Tom20 and MnSOD are physically associated with each other (Fig. 5). These data indicate that Tom 20 is indispensable in monensin-induced increase of mitochondrial MnSOD protein in HEK293 cells. This could also be a molecular mechanism for severe EAE-induced increase in mitochondrial MnSOD protein level in the mouse kidney cortex.

\section{$\mathrm{Na}, \mathrm{K}-\mathrm{ATPase}$ activity and mitochondrial ROS contribute to monensin-induced increases of mitochondrial SGK1, which then leads to increases of mitochondrial Tom 20 and MnSOD protein abundance}

SGK1 is a positive regulator of Na,K-ATPase. Expression of SGK1 increases Na,K-ATPase activity [19]. We found that ouabain reduces monensin-induced increase in the mitochondrial SGK1 protein level (Fig. 3), suggesting that $\mathrm{Na}, \mathrm{K}$-ATPase contributes to the effect of monensin on mitochondrial SGK1 and a positive feedback loop between Na,KATPase and SGK1. SGK1 is also regulated by ROS, as the antioxidant tempol inhibits aldosterone-induced increases of ROS and SGK1 mRNA abundance in vitro in the cultured rat peritoneal fibroblasts [31] and high salt diet-induced ROS and SGK1 mRNA levels in the rat glomeruli in vivo [16]. We found that catalase reduces monensin-induced increase of mitochondrial SGK1 protein levels (Fig. 3). Since ouabain and catalase inhibit monensin-induced increases of mitochondrial ROS [25], we interpret our findings as that mitochondrial ROS resulted from increased Na,K-ATPase activity mediate the effect of monensin on the mitochondrial SGK1. It used to be believed that SGK1 was only present in the cytosol [1]. It was later found that SGK1 is also present in mitochondria [2] and the outer mitochondrial membrane [5]. The present studies demonstrate that mitochondrial SGK1 protein abundance is increased in response to increases of Na,K-ATPase activity and mitochondrial ROS, whereas the cytosolic SGK1 is not (Fig. 3b), indicating that SGK1 in these two compartments is regulated by different mechanisms.

Severe EAE increases mitochondrial ROS, SGK1, Tom20 and MnSOD protein abundance (Figs. 1 and 3) [25, 35].
It is understandable that an increase in mitochondrial ROS would increase mitochondrial MnSOD to protect mitochondria from the oxidant-induced damage. However, the exact molecular mechanism remains incompletely understood. We argue that SGK1 could relay the effect of mitochondrial ROS on mitochondrial MnSOD by increasing mitochondrial Tom 20 protein levels in the kidney cortex, as GSK650394 attenuates the effect of monensin on the mitochondrial Tom20 and MnSOD protein abundance (Fig. 4a, b). The proposed mechanism may also explain the observations that overexpression of SGK1 decreases ROS, alleviates mitochondrial dysfunction, and rescues cell death induced by 6-hydroxydopamine in vitro and in vivo [12].

Despite the importance of protein targeting to mitochondria, and sorting to distinct mitochondrial subcompartments, the mitochondrial protein importation mechanism has not been well studied. Knowledge about how Tom20 is regulated is sparse. We found that monensin increases mitochondrial Tom 20 protein levels, and this effect is inhibited by ouabain, catalase and GSK650394, suggesting that Na,KATPase, mitochondrial ROS and SGK1 regulate mitochondrial Tom 20 protein expression. Whether SGK1 regulates Tom 20 through phosphorylation either directly or indirectly remains to be determined.

In summary, severe EAE increases mitochondrial SGK1 and Tom 20 protein abundance and has no significant effect on their mRNA levels in the mouse kidney cortex, the same results are found with monensin in HEK293 cells. Catalase and ouabain inhibit monensin-induced increase of mitochondrial SGK1 and Tom 20 protein abundance. Inhibition of SGK1 by GSK650394 attenuates monensin-induced increases of mitochondrial Tom20 and MnSOD protein abundance. Further, Tom 20 and MnSOD physically associate with each other, and siRNA-mediated knockdown of Tom20 reduces monensin-induced increase of mitochondrial MnSOD protein abundance. Based on our previous observations that severe EAE increases Na,K-ATPase activity, mitochondrial Complex II, Complex VI, ROS and MnSOD activities and mitochondrial MnSOD protein abundance in the kidney cortex, and that monensin shows similar effects in HEK293 cells, and ouabain and catalase inhibit monensininduced mitochondrial ROS and MnSOD protein abundance [25, 35], we propose a model as outlined in Fig. 6. Activation of $\mathrm{Na}, \mathrm{K}$-ATPase leads to an increase in mitochondrial function to meet the energy demand for the increased $\mathrm{Na}$,K-ATPase activity. Increased mitochondrial respiration results in stimulation of mitochondrial production of ROS as a by-product, which leads to augmentation of mitochondrial SGK1. SGK1 then increases mitochondrial Tom 20 protein abundance, resulting in the importation of more MnSOD to the mitochondrial matrix to protect mitochondria from mitochondrial ROS-induced harm. 


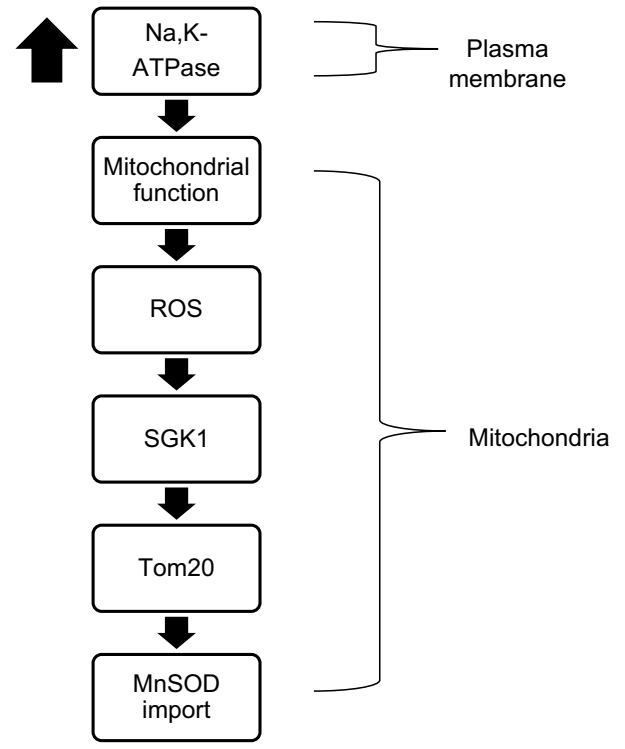

Fig. 6 Schematic presentation of how activation of Na,K-ATPase increases the mitochondrial MnSOD protein abundance in HEK293 cells

Acknowledgements This work was funded in part by the grant (MED83-3923) from the collaborative health initiative research program between the National Heart Lung and Blood Institute and the Henry Jackson Foundation for the Advancement of Military Medicine, the National Multiple Sclerosis Society Grant PP3448 and a Uniformed Services University education grant. The authors thank Dr. Louis Pangaro for his help in obtaining the education grant.

Disclaimer The content and views expressed in this article are the sole responsibility of the authors and do not necessarily reflect the views or policies of the Department of Defense or US Government. Mention of trade names, commercial products, or organizations does not imply endorsement by the Department of Defense or US Government.

\section{References}

1. Alvarez de la Rosa D, Coric T, Todorovic N, Shao D, Wang T, Canessa CM (2003) Distribution and regulation of expression of serum- and glucocorticoid-induced kinase-1 in the rat kidney. $\mathrm{J}$ Physiol 551:455-466

2. Cordas E, Naray-Fejes-Toth A, Fejes-Toth G (2007) Subcellular location of serum- and glucocorticoid-induced kinase- 1 in renal and mammary epithelial cells. Am J Physiol Cell Physiol 292:C1971-1981

3. Dudek J, Rehling P, van der Laan M (2013) Mitochondrial protein import: common principles and physiological networks. Biochim Biophys Acta 1833:274-285

4. Efendiev R, Bertorello AM, Zandomeni R, Cinelli AR, Pedemonte $\mathrm{CH}$ (2002) Agonist-dependent regulation of renal $\mathrm{Na}^{+}$, $\mathrm{K}^{+}$-ATPase activity is modulated by intracellular sodium concentration. J Biol Chem 277:11489-11496

5. Engelsberg A, Kobelt F, Kuhl D (2006) The N-terminus of the serum- and glucocorticoid-inducible kinase Sgk1 specifies mitochondrial localization and rapid turnover. Biochem J 399:69-76
6. Ferraris JD, Williams CK, Persaud P, Zhang Z, Chen Y, Burg MB (2002) Activity of the TonEBP/OREBP transactivation domain varies directly with extracellular $\mathrm{NaCl}$ concentration. Proc Natl Acad Sci USA 99:739-744

7. Gerbeth C, Schmidt O, Rao S, Harbauer AB, Mikropoulou D, Opalinska M, Guiard B, Pfanner N, Meisinger C (2013) Glucose-induced regulation of protein import receptor Tom22 by cytosolic and mitochondria-bound kinases. Cell Metab 18:578-587

8. Grey JY, Connor MK, Gordon JW, Yano M, Mori M, Hood DA (2000) Tom20-mediated mitochondrial protein import in muscle cells during differentiation. Am J Physiol Cell Physiol 279:C1393-1400

9. Guffanti AA, Cohn DE, Kaback HR, Krulwich TA (1981) Relationship between the $\mathrm{Na}^{+} / \mathrm{H}^{+}$antiporter and $\mathrm{Na}^{+} /$substrate symport in Bacillus alcalophilus. Proc Natl Acad Sci USA 78:1481-1484

10. Hucke S, Eschborn M, Liebmann M, Herold M, Freise N, Engbers A, Ehling P, Meuth SG, Roth J, Kuhlmann T, Wiendl H, Klotz L (2016) Sodium chloride promotes pro-inflammatory macrophage polarization thereby aggravating CNS autoimmunity. J Autoimmun 67:90-101

11. Ip WK, Medzhitov R (2015) Macrophages monitor tissue osmolarity and induce inflammatory response through NLRP3 and NLRC4 inflammasome activation. Nat Commun 6:6931

12. Iqbal S, Howard S, LoGrasso PV (2015) Serum- and glucocorticoid-inducible kinase 1 confers protection in cell-based and in in vivo neurotoxin models via the c-Jun $\mathrm{N}$-terminal kinase signaling pathway. Mol Cell Biol 35:1992-2006

13. Jorg S, Kissel J, Manzel A, Kleinewietfeld M, Haghikia A, Gold R, Muller DN, Linker RA (2016) High salt drives Th17 responses in experimental autoimmune encephalomyelitis without impacting myeloid dendritic cells. Exp Neurol 279:212-222

14. Kaltimbacher V, Bonnet C, Lecoeuvre G, Forster V, Sahel JA, Corral-Debrinski M (2006) mRNA localization to the mitochondrial surface allows the efficient translocation inside the organelle of a nuclear recoded ATP6 protein. RNA 12:1408-1417

15. Kempson S, Thompson N, Pezzuto L, Glenn Bohlen H (2007) Nitric oxide production by mouse renal tubules can be increased by a sodium-dependent mechanism. Nitric Oxide 17:33-43

16. Kitada K, Nakano D, Liu Y, Fujisawa Y, Hitomi H, Shibayama Y, Shibata H, Nagai Y, Mori H, Masaki T, Kobori H, Nishiyama A (2012) Oxidative stress-induced glomerular mineralocorticoid receptor activation limits the benefit of salt reduction in Dahl saltsensitive rats. PLoS One 7:e41896

17. Kleinewietfeld M, Manzel A, Titze J, Kvakan H, Yosef N, Linker RA, Muller DN, Hafler DA (2013) Sodium chloride drives autoimmune disease by the induction of pathogenic TH17 cells. Nature 496:518-522

18. Krementsov DN, Case LK, Hickey WF, Teuscher C (2015) Exacerbation of autoimmune neuroinflammation by dietary sodium is genetically controlled and sex specific. FASEB J 29:3446-3457

19. Lang F, Bohmer C, Palmada M, Seebohm G, Strutz-Seebohm N, Vallon V (2006) (Patho)physiological significance of the serum- and glucocorticoid-inducible kinase isoforms. Physiol Rev 86:1151-1178

20. Li Y, Huang TT, Carlson EJ, Melov S, Ursell PC, Olson JL, Noble LJ, Yoshimura MP, Berger C, Chan PH, Wallace DC, Epstein CJ (1995) Dilated cardiomyopathy and neonatal lethality in mutant mice lacking manganese superoxide dismutase. Nat Genet 11:376-381

21. Li Y, Reuter NP, Li X, Liu Q, Zhang J, Martin RC (2010) Colocalization of MnSOD expression in response to oxidative stress. Mol Carcinog 49:44-53

22. Luk E, Yang M, Jensen LT, Bourbonnais Y, Culotta VC (2005) Manganese activation of superoxide dismutase 2 in the mitochondria of Saccharomyces cerevisiae. J Biol Chem 280:22715-22720 
23. Millar DG, Shore GC (1996) Signal anchor sequence insertion into the outer mitochondrial membrane. Comparison with porin and the matrix protein targeting pathway. J Biol Chem 271:25823-25829

24. Miranda PM, De Palma G, Serkis V, Lu J, Louis-Auguste MP, McCarville JL, Verdu EF, Collins SM, Bercik P (2018) High salt diet exacerbates colitis in mice by decreasing Lactobacillus levels and butyrate production. Microbiome 6:57

25. Packialakshmi B, Zhou X (2018) Experimental autoimmune encephalomyelitis (EAE) up-regulates the mitochondrial activity and manganese superoxide dismutase (MnSOD) in the mouse renal cortex. PLoS One 13:e0196277

26. Pao AC (2012) SGK regulation of renal sodium transport. Curr Opin Nephrol Hypertens 21:534-540

27. Sherk AB, Frigo DE, Schnackenberg CG, Bray JD, Laping NJ, Trizna W, Hammond M, Patterson JR, Thompson SK, Kazmin D, Norris JD, McDonnell DP (2008) Development of a smallmolecule serum- and glucocorticoid-regulated kinase-1 antagonist and its evaluation as a prostate cancer therapeutic. Cancer Res 68:7475-7483

28. Sundstrom B, Johansson I, Rantapaa-Dahlqvist S (2015) Interaction between dietary sodium and smoking increases the risk for rheumatoid arthritis: results from a nested case-control study. Rheumatology (Oxford) 54:487-493

29. Wispe JR, Clark JC, Burhans MS, Kropp KE, Korfhagen TR, Whitsett JA (1989) Synthesis and processing of the precursor for human mangano-superoxide dismutase. Biochim Biophys Acta 994:30-36

30. Wu C, Yosef N, Thalhamer T, Zhu C, Xiao S, Kishi Y, Regev A, Kuchroo VK (2013) Induction of pathogenic TH17 cells by inducible salt-sensing kinase SGK1. Nature 496:513-517
31. Yamahara H, Kishimoto N, Nakata M, Okazaki A, Kimura T, Sonomura K, Matsuoka E, Shiotsu Y, Adachi T, Matsubara H, Iwasaka T, Mori Y (2009) Direct aldosterone action as a profibrotic factor via ROS-mediated SGK1 in peritoneal fibroblasts. Kidney Blood Press Res 32:185-193

32. Yang X, Yao G, Chen W, Tang X, Feng X, Sun L (2015) Exacerbation of lupus nephritis by high sodium chloride related to activation of SGK1 pathway. Int Immunopharmacol 29:568-573

33. Zhang Y, Baaden M, Yan J, Shao J, Qiu S, Wu Y, Ding Y (2010) The molecular recognition mechanism for superoxide dismutase presequence binding to the mitochondrial protein import receptor Tom20 from Oryza sativa involves an LRTLA motif. J Phys Chem B 114:13839-13846

34. Zhou X, Gallazzini M, Burg MB, Ferraris JD (2010) Contribution of SHP-1 protein tyrosine phosphatase to osmotic regulation of the transcription factor TonEBP/OREBP. Proc Natl Acad Sci USA 107:7072-7077

35. Zhou X, Packialakshmi B, Xiao Y, Nurmukhambetova S, Lees JR (2017) Progression of experimental autoimmune encephalomyelitis is associated with up-regulation of major sodium transporters in the mouse kidney cortex under a normal salt diet. Cell Immunol $317: 18-25$

Publisher's Note Springer Nature remains neutral with regard to jurisdictional claims in published maps and institutional affiliations. 